\title{
Recent Advances in Systematic and Targeted Prostate Biopsies
}

\author{
Konstantinos Devetzis \\ Francesca Kum (D) ${ }^{1,2}$ \\ Richard Popert ${ }^{2}$ \\ 'King's College School of Medicine, \\ London, UK; ${ }^{2}$ Department of Urology, I ${ }^{\text {st }}$ \\ Floor Southwark Wing, Guy's Hospital, \\ London, SEI 9RT, UK
}

\begin{abstract}
Prostate biopsy is the definitive investigation to diagnose prostate cancer. The ideal procedure would be one that offers fast and efficient results safely as an outpatient procedure. Historically, transrectal ultrasound (TRUS) biopsy is considered the gold standard but transrectal biopsy can under-sample the anterior and apical regions of the prostate and is associated with a risk of prostate biopsy-related sepsis, which may require intensive care admission. Transperineal (TP) biopsy addresses the inefficient sampling of TRUS biopsy but historically has been done under general anaesthetic, which makes it difficult to incorporate into timed diagnostic pathways such as the National Health Service (NHS) 2-week cancer pathway. TRUS biopsy has remained the mainstay of clinical diagnosis because of its simplicity; however, the recent development of simpler local anaesthetic transperineal techniques has transformed outpatient biopsy practice. These techniques practically eliminate prostate biopsy-related sepsis, have a shallow learning curve and offer effective sampling of all areas of the prostate in an outpatient setting. The effectiveness of TP biopsy has been enhanced by the introduction of multiparametric MRI prior to biopsy, the use of PSA density for risk stratification in equivocal cases and combined with more efficient targeted and systematic biopsies techniques, such as the Ginsburg Protocol, has improved the tolerability and diagnostic yield of local anaesthetic TP biopsies, reducing the risk of complications from the oversampling associated with transperineal template mapping biopsies. Areas where the literature remains unclear is the optimum number of cores needed to detect clinically significant disease (CSD) in patients with a definable lesion on MRI, in particular, whether there is a need for systematic biopsy in the face of equivocal MRI findings to ensure no CSD is missed. The Covid-19 pandemic has had a profound impact on prostate cancer referrals and prostate biopsy techniques within the UK; prior to the pandemic $65 \%$ of all prostate biopsies were TRUS, since the pandemic the proportions have reversed such that now over $65 \%$ of all prostate biopsies in the NHS are transperineal.
\end{abstract}

Keywords: transperineal, transrectal, local anaesthetic, prostate biopsies, Covid-19

\section{Introduction}

Prostate biopsy is the mainstay of prostate cancer diagnosis. Biopsy techniques are constantly evaluated and revised to ensure accurate detection of clinically significant disease (CSD), while using a fast and efficient technique. ${ }^{1}$ Transrectal ultrasound guided (TRUS) biopsy was once the gold standard as it was quick and easy, but it does not allow access to the whole of the peripheral zone (PZ) and anterior prostate and is accompanied by a risk of sepsis. Transperineal (TP) biopsy was developed over the last 20 years, but historically has required complex equipment, multiple cores were taken (Template Mapping Biopsy) and general anaesthesia (GA) was needed. ${ }^{2}$ Within the UK National Health Service (NHS), the introduction of pre-biopsy MRI has
Correspondence: Richard Popert Department of Urology, I It Floor Southwark Wing, Guy's Hospital, St Thomas' St, London, SEI 9RT, UK Tel +44 207I887I88

Email rick.popert@gstt.nhs.uk 
transformed the diagnostic pathway, reducing unnecessary prostate biopsies and improving the detection of CSD, and has paved the way for the development of outpatient targeted and systematic TP biopsy techniques replacing the routine use of Transrectal prostate biopsy. This has become known as the "TRexit" Initiative. ${ }^{3}$

\section{Materials and Methods}

A literature search of peer-reviewed articles was performed on the topics of the history, recent changes and advances in the field of prostate biopsies. The Medical Subject Headings used were "Prostate biopsy", "Transperineal", "Freehand", "Systematic", "Targeted", "Cores", "MRI prostate biopsy", "Covid-19". The articles were evaluated and selected based on author credentials, adherence to current guidelines and use of up-to-date techniques. Any relevant data will be presented and discussed, always with regards to its reliability.

\section{History of Prostate Biopsies}

Historically prostate biopsy was carried out transperineally but the introduction of transrectal ultrasound in the 1980s changed prostate biopsy practice dramatically. Although TP biopsy was preferred to transrectal biopsy due to concerns regarding faecal contamination, Hodge et al.'s sextant method of TRUS biopsy overshadowed preceding methods of random biopsy and established a new standard. ${ }^{4}$ Systematic TRUS biopsy was compared with a targeted TRUS biopsy of palpable or ultrasound visible abnormalities using a spring-loaded biopsy gun. The apex, middle and base of each lobe of the prostate were systematically sampled. With a cancer detection rate of $62 \%$, the systematic biopsy detected $9 \%$ of disease missed by targeted only. As a result, the sextant method became the gold standard. However, it is important to note this was a high-risk group of patients with palpable abnormalities but historically, this paper was important as it set a new standard, in a time where there was no common consensus regarding prostate biopsy techniques. ${ }^{4}$

The 6-core Systematic TRUS biopsy had limitations which were addressed by the work of Joseph Presti et al., who advocated that a 10-core systematic TRUS biopsy should include lateral Peripheral Zone cores in addition to the sextant method, which increased cancer detection by $20-35 \%{ }^{5,6}$ However, the anterior prostate and apical area remained undersampled by the TRUS biopsy approach. This limitation combined with the risk of Prostate BiopsyRelated Sepsis (PBRS) led to the reappearance of TP

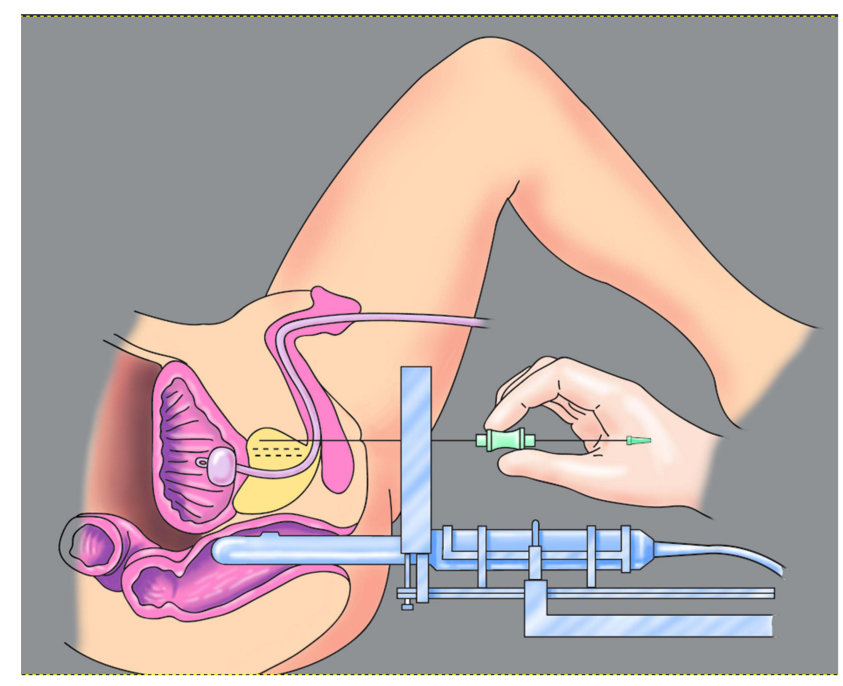

Figure I Transperineal template biopsy with brachytherapy grid. Image courtesy from BXTA.

biopsy in clinical practice, facilitated by the introduction of prostate brachytherapy as a transperineal intervention. The transperineal approach for biopsy offered better sampling of the anterior and apical area of the prostate, detected CSD that was previously missed, and also came with significantly lower risk of PBRS, a potentially lifethreatening complication of TRUS biopsy. ${ }^{7-10}$

Amongst many treatment changes introduced over the years has been the development of focal therapy as a prostate cancer treatment modality; before MRI scans became routinely available this required a more accurate localisation of lesions which was addressed using a brachytherapy grid. ${ }^{11}$ The template was fixed and allowed for mapping of the prostate in three dimensions (Figure 1) and rightfully earned its place in clinical practice in many centres worldwide. Nakai et al. showed that the method was effective at both detecting presence of disease as well as CSD with a number of cores ranging from 18 to $75 .{ }^{12}$ As expected systematic biopsy of the prostate was taken from every hole in the grid, with a $5 \mathrm{~mm}$ grid being statistically more efficient at detecting cancer than a $10 \mathrm{~mm}$ interval $(95 \%$ and $78 \%$, respectively) while also detecting more $\mathrm{CSD}$, as shown by Crawford et al. However, these numbers were at the cost of a greater number of biopsies and consequently greater complications, particularly urinary retention. ${ }^{13}$

\section{The Ginsburg Systematic TP Biopsy Protocol}

Despite its high detection rate of prostate cancer, the template technique presented with post-procedural 
complications, with an incidence of $8.3 \%$ of haematuria and a risk of urinary retention in $11-13 \%$ of patients. $^{14}$ Independent of the size of the prostate an increased number of cores, specifically more than 35, increased the risk of urinary retention. ${ }^{15}$ This issue was addressed by the Ginsburg Consensus Meeting on Transperineal Biopsies which was held in Heidelberg by a group of urologists who were interested in standardising TP biopsies to help evaluate MRI abnormalities and deliver more effective systematic biopsy. The Ginsburg Study Group by consensus adopted a 24-38 Core systematic biopsy protocol, which became known as the Ginsburg TP Biopsy Protocol. ${ }^{16}$

The Ginsburg Protocol preferentially targets the PZ and divides it into anterior, mid and posterior sectors, while avoiding the transition and periurethral zones (Figure 2). The rationale behind this approach is to take advantage of the fact that the majority of prostate carcinomas are in the PZ and that glandular oedema around the urethra can be reduced by not taking so many cores from the transition zone in which the incidence of isolated prostate cancer is less than $4 \%$. Importantly, the reduced number of cores did not negatively affect the cancer detection rate. ${ }^{16-18}$

This systematic biopsy approach was originally developed and described at Guy's Hospital as Transperineal Sector Biopsies but it was decided at the Consensus that no one individual or institution should take credit and so it was renamed as the Ginsburg Protocol (Personal Communication R Popert Consultant Urologist, GSTT). Vyas et al. performed biplanar US-guided TP biopsy on 634 patients with a previously negative TRUS biopsy, primary biopsies or low-risk disease diagnosed after 12-core TRUS biopsy from 2007 to 2011, with a number of cores ranging from $24-38$; if the size was $>30 \mathrm{~mL}$, basal cores were taken in addition to the scheme. Only $1.7 \%$ had acute urinary retention and no cases of urosepsis were reported. There was no statistical significance between the number of previous negative TRUS biopsy and the chance of a positive sector TP biopsy, most likely because TP biopsy offers sampling of areas of the prostate that were not sampled in TRUS biopsy. ${ }^{18}$ Eldred-Evans et al. reviewed the sector method on biopsy naïve patients, selected based on elevated PSA and/or abnormal digital rectal examination (DRE). The detection rate was found to be $61.9 \%$, compared with 40 $44 \%$ of TRUS biopsy for patients with the same selection criteria, with $75.1 \%$ of the cancers being CSD. No patients developed urosepsis and the urinary retention rate was only $1.3 \%$ without routine prescription of $\alpha$-blockers. The study also identified $17.3 \%$ of cancers exclusively located in the anterior part of the prostate, potentially missed by TRUS biopsy. $^{20}$ The Ginsburg Protocol has been increasingly adopted as an alternative to Template Mapping biopsy and the protocol can be adapted to systematic TP biopsy taken using any method from template, to freehand and to MRI/ TRUS fusion.

\section{PSA-Density}

With factors such as family history, prostate-specific antigen density (PSAD) and pre-biopsy multiparametric MRI (mpMRI) risk stratification is much better for patients suspected to have prostate cancer. The clinical application of PSA as a cancer marker was introduced by Stamey in the 1980s but its correlation with the size of the prostate with ageing complicated its use in risk stratification. ${ }^{21}$ To minimise unnecessary biopsies of non-CSD, PSAD was introduced, the ratio of PSA serum levels over the volume of the prostate. MacAskill et al., using systematic TP biopsy as reference, found that PSAD calculated from MRI (MR-PSAD) is superior to PSA alone, but as MR-

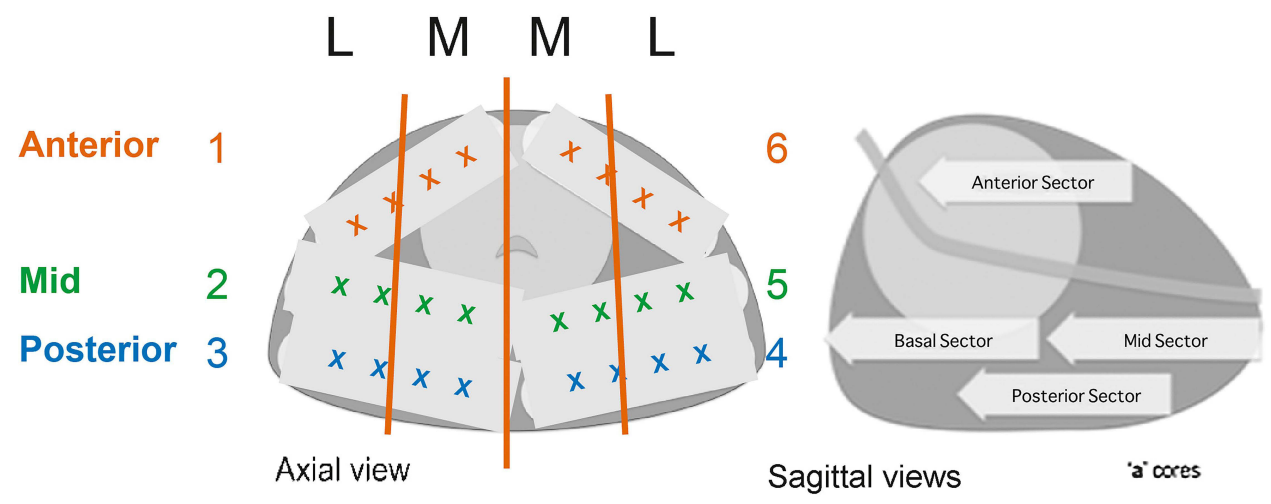

Figure 2 Ginsburg Protocol template. Adapted from Eur Urol, Hansen N, Patruno G, Wadhwa K, et al. Magnetic Resonance and Ultrasound Image Fusion supported transperineal prostate biopsy using the ginsburg protocol: technique, learning points, and biopsy results. 332-340, Copyright (2016), with permission from Elsevier. ${ }^{19}$ 
PSAD cut-off values rise, more CSD is missed. ${ }^{22}$ Washino et al. combined MR-PSAD with the imaging findings of mpMRI; two high-risk groups were observed: PI-RADS $\geq 4$ with MR-PSAD $\geq 0.15$ and PI-RADS 3 with MR-PSAD $\geq 0.30$ were associated with the highest CSD detection rate (76-97\%). ${ }^{23}$ A PSAD $\geq 0.15 \mathrm{ng} / \mathrm{ml} / \mathrm{cc}$ presented with clinically non-significant disease, increasing the negative predictive value $(91.15 \%) .{ }^{24}$ PSAD is an important factor in the selection of patients for prostate biopsy where the MRI findings are equivocal. Since there is not much research on equivocal MRI, Sonmez et al. investigated patients with PI-RADS 3 and PSA $<10 \mathrm{ng} / \mathrm{ml}$ and suggest a cut-off PSAD value of 0.11 and below to preclude CSD. ${ }^{25}$ With a PSAD $<0.1 \mathrm{ng} / \mathrm{ml} / \mathrm{cc}$ and a normal MRI the risk of CSD is so low that most patients can be reassured and discharged for PSA observation.

\section{MRI \& Prostate Biopsy}

Incorporation of mpMRI in the prostate cancer diagnosis pathway has been responsible for transforming the prostate biopsy pathway such that pre-biopsy MRI has been universally adopted across the NHS.

\section{In-Bore MRI}

MRI guidance can be used in the prostate biopsy technique itself. In-bore MRI biopsy involves taking the biopsy using real-time MRI. The technique is promising due to its high detection rate and minimal complications. However, it has not been greatly adopted due to the complexity of the procedure, the cost and more importantly the inability to perform the biopsy in an outpatient setting. Furthermore, there are limitations to the number of biopsies that can be taken. ${ }^{26,27}$

\section{Pre-Biopsy MRI}

A number of studies have demonstrated the efficacy of using mpMRI prior to biopsy. Porpiglia et al. randomly allocated biopsy naive patients to Group A, with mpMRI prior to biopsy and receiving targeted biopsy for visible lesions or systematic biopsy in the absence of visible lesions, and Group B, receiving only systematic biopsy. The detection rate of CSD (Gleason score $\geq 7$ or Maximum Cancer Core Length of $5 \mathrm{~mm}$ ) was $87.0 \%{ }^{28}$ In 2017 , the multi-centre PROMIS study demonstrated that TRUS biopsy missed $52 \%$ of CSD detected by TP biopsy and that mpMRI can reduce the number of unnecessary biopsies by $27 \%$. A sensitivity of $93 \%$ compared with $48 \%$ for TRUS was impressive, whilst the high negative predictive value (NPV) was reassuring. ${ }^{29}$ A literature review by Ahmed et al. concluded that mpMRI prior to biopsy is not only efficient but also highly cost-effective when weighed against the cost of unnecessary biopsies as well as unnecessary surveillance and subsequent treatment of non-CSD. ${ }^{30}$ The PRECISION study in 2018, established the superiority of mpMRI targeted biopsies compared with 10-12 core systematic prostate biopsy, with higher efficacy for detecting CSD and less insignificant disease. ${ }^{31}$ Similar to the other MRI-based studies, they only performed a targeted TP biopsy of MRI visible lesions, but most studies suggest there continues to be a need for systematic biopsies in addition to targeted biopsy. ${ }^{19}$

\section{Cognitive vs MRI Fusion}

Cognitive biopsies require the operator to review the patient's MRI and have a mental image of the target, whereas MRI-US fusion involves marking of the lesion on mpMRI and specialised software fusion with real time US images, to improve the localisation of the lesion (see Table 1).

BiopSee was the first reported Transperineal MRI-US fusion system in 2011, with the ability to save the outlines for each patient (useful for active surveillance patients' follow up). The template reassures safe position of the needle even with readjusting movements of the patient, however, it limits the number of cores taken from the anterior part of large prostates. A total of 807 patients underwent fusion TP biopsy with BiopSee (24-core Ginsburg Protocol used for systematic biopsy and 2 core targeted biopsies) in a multi-centre study by Hansen et al. The detection rate was $68 \%$ with CSD rate being $49 \%{ }^{19,32}$

Esaote is another option featuring adjustment of the transparency between ultrasound and MRI, but most importantly allowing the biopsy to be taken using one perineal access point, thus allowing sampling of the peripheries of

Table I MRI-US Fusion Biopsy Systems Currently in Clinical Use

\begin{tabular}{|l|l|}
\hline Type of Biopsy & Use of Biopsy \\
\hline $\begin{array}{l}\text { TRUS biopsy with } \\
\text { MRI-US Fusion }\end{array}$ & The GPS sensor allows TRUS biopsy use \\
\hline $\begin{array}{l}\text { TP biopsy with MRI- } \\
\text { US Fusion }\end{array}$ & $\begin{array}{l}\text { The probe is on a stepping unit with } \\
\text { a brachytherapy grid which is linked directly } \\
\text { to the fusion system }\end{array}$ \\
\hline $\begin{array}{l}\text { Freehand TP-MRI-US } \\
\text { Fusion }\end{array}$ & The GPS allows it to be used freehand \\
\hline
\end{tabular}


large prostate glands. However, the landmarks are set by the operator themselves and not by the system's detection of overlapping structures on ultrasound and MRI; this can lead to standardisation errors. ${ }^{33}$ In 2020, Marra et al. investigated the efficacy and tolerability of freehand mpMRI fusion-targeted biopsy on 459 men. Systematic biopsy involved taking 12 cores from the PZ but not routinely from the anterior and transition zones. The fusion system yielded good results with $63.6 \%$ detection rate. CSD was $53.8 \%$ in the fusion-targeted group and $45 \%$ in the systematic group. The process caused low-level pain, retention presented in only 3 cases with suspected BPH; and no UTI or urosepsis episodes were recorded. ${ }^{34,35}$

Even though fusion biopsies are promising there appears to be no clear advantage in detection rate compared with cognitive biopsies, ${ }^{36}$ as these methods show comparable results and no statistically significant difference. ${ }^{37}$ Looking at similar sample sizes of two different studies, Zhang et al.'s freehand Esaote fusion TP biopsy ${ }^{38}$ and Neale et al.'s freehand PrecisionPoint cognitive MRI TP biopsy, ${ }^{39}$ cognitive MRI biopsy shows better detection rate $(84 \%$ compared with 50.45\%) and CSD detection (69\% compared with $44.2 \%$ ). Nevertheless, Oderda et al., comparing the two methods with 50 patients randomised into KOELIS fusion or cognitive biopsy, suggest that fusion may have a role in better detection of prostate cancer for lesions $\leq 10 \mathrm{~mm} .{ }^{40}$ Due to its high cost, fusion biopsy might be better reserved for hard to target smaller lesions with negative cognitive targeted biopsies.

\section{US \& Prostate Biopsy}

Routine pre-biopsy MRI in all patients, such as occurs within the NHS, is unusual. This is due to a combination of perceived cost and the need for specialist uroradiologists for reporting; mpMRI is not an available modality in many institutions across the world. Consequently, in centres where there is limited access to mpMRI or specialist reporting, the use of advances in ultrasound has been drawing attention as a potential, more affordable and thus more accessible diagnostic imaging compared with mpMRI. One of the first and most popular diagnostic methods has been HistoScanning, a software based system that uses backscatter signals normally omitted by normal ultrasound to provide a 3D image and detect possible lesions. Prostatic HistoScanning True Targeting (PHS-TT) offers fusion of the HistoScanning imaging with live ultrasound. Although initial outcomes reported were promising, with a 100\% sensitivity and
$100 \% \mathrm{NPV},{ }^{41,42}$ the lack of reproducibility by subsequent studies and the decrease in these values as the sample size increased should be appraised. ${ }^{43}$ Nunez-Mora et al., with a small sample size, found a NPV of $96.5 \%$. Schiffmann et al. has been the largest study to investigate the application of HistoScanning in prostate biopsy. They demonstrated a low NPV of 28.8-33.1\% and a high false positive rate of $73.1 \%{ }^{44}$ Other new ultrasound modalities exist, including Artificial Neural Network Analysis Computed Transrectal ultrasound (ANNA/C-TRUS), Shear-Wave Elastography (SWE) and Contrast-Enhanced Ultrasound (CEUS). Despite their high sensitivity and specificity values, the results from large-scale clinical trials that could provide a clearer picture of their effectiveness in prostate biopsy, in comparison to the available evidence on MRI in the literature, are still pending. ${ }^{45}$ The major dilemma lies in the operator-dependent nature of these ultrasound modalities as well as identified uncontrolled factors, including prostate size and distance of probe from the anterior prostatic capsule. At present, the role of these modalities, especially in light of the abundant evidence of the superiority of mpMRI, remains doubtful. ${ }^{46}$

\section{Freehand Transperineal Biopsies}

Transperineal template mapping and Ginsburg Protocol biopsies using a brachytherapy stepping unit require complex equipment and a general anaesthetic which make them impractical for general use. However, increasingly urologists are resorting to other means to target the prostate transperineally. One of the most important milestones in the history of prostate biopsy has been the use of local anaesthetic freehand biopsy. As the name suggests, the needle can be handled manually, going through the perineal skin under local anaesthetic (LA) in an outpatient setting. A total of 1287 freehand transperineal biopsies were performed by Stefanova et al. with a detection rate of $49.8 \%$ and CSD rate of $60.1 \%$, suggesting an increase in detection due to better sampling of the anterior zone compared with TRUS biopsy. With a minimum of 10 cores, including the anterior and posterior apex, anterior and posterior base and mid lateral left and right sides, there were $1.6 \%$ cases of acute urinary retention and only $0.3 \%$ of post-procedure infection. No cases of urosepsis were reported in any of the patients. ${ }^{47}$ These results are comparable to reports of TP Sector biopsies using the Ginsburg Protocol but importantly were achieved under local anaesthetic. ${ }^{18-20}$ Utilising a VAS (visual analogue scale) for pain they found that 3.1/10 was the highest pain reported and that was during anaesthetic 
administration. When 70 patients were asked about the difference in discomfort between TRUS biopsy and freehand TP biopsy there was an equivocal response, with most reporting comparable discomfort. ${ }^{47}$

\section{PrecisionPoint $^{\mathrm{TM}}$ Transperineal Access System}

The major problem with freehand biopsies is there is not actually a free hand. One hand holds the ultrasound probe, the other holds the biopsy gun and it is necessary to align the biopsy needle with the ultrasound array. This can be difficult to achieve and is difficult to learn, which limits the generalizability. The biopsies tend to be taken using a "fan" distribution and this can result in undersampling of the anterior apical prostate. The problem with alignment of the probe with the biopsy guide has been overcome using a simple disposable device, the PrecisionPoint $^{\mathrm{TM}}$ Transperineal Access System. Unlike other methods involving piercing the perineal skin multiple times to reach the prostate longitudinally, the PrecisionPoint uses two access points in the perineum for each side. The 5-aperture grid and the stabilization unit are attached directly onto the shaft of the transrectal ultrasound probe (Figure 3). The manipulation of the access needle is not limited by the pelvic bones and its mechanism ensures the biopsy needle is in line with the ultrasound probe and so always remains visible. The 5 holes in the guide allow the operator to access different areas of the prostate, particularly the anterior apical area. Cognitive targeting is achieved easily by simply directing the access needle at the region of interest through the most appropriate hole on the grid. The PrecisionPoint system

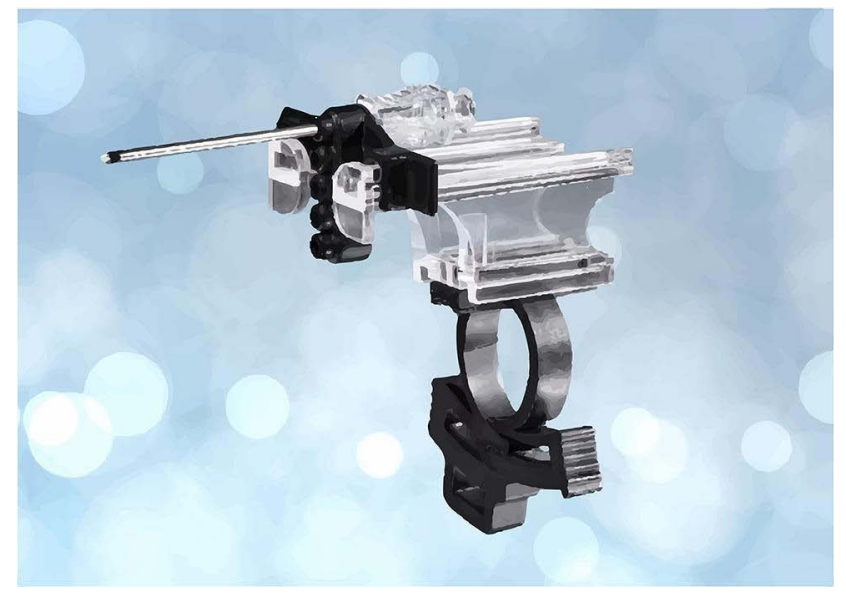

Figure 3 PrecisionPoint. Image courtesy with permission from Perineologic (https://perineologic.com/precisionpoint/) $^{49}$ has been shown to be well tolerated under LA with equivalent cancer detection rates to standard transperineal biopsies under general anaesthetic. In common with TP biopsies sepsis rates are almost zero, and unlike TRUS biopsy this can be achieved without routine antibiotics. Rates of urinary retention under local anaesthetic using the limited perineal puncture approach are less than $0.5 \%$. Importantly, urology advanced nurse practitioners and radiographers can be trained to use the PrecisionPoint techniques, thus distributing the workload between more healthcare staff. ${ }^{10,48}$

Kum et al. presented the initial outcomes of PrecisionPoint for 176 men in the UK. They found a high detection rate of $79 \%, 76.3 \%$ for biopsy naive patients. PrecisionPoint seems to be good at detecting CSD $(60.9 \%)$ while avoiding overdiagnosis of patients who would not benefit from treatment. The use of VAS scores (out of $100 \mathrm{~mm}$ ) yielded low pain generally with the highest being recorded during administration of the LA, as reported from other studies as well. Interestingly they found that the mean pain was $27.5 / 100$ when the procedure was performed in the outpatient clinic and 45/100 when done in the daysurgery unit, demonstrating how patient expectations may affect their perception of pain with the increased anxiety associated with hospital admission and peri-operative processes. No cases of PBRS were reported. Self-limiting haematuria and haematospermia in $75.4 \%$ and $40.4 \%$, respectively were reported with only $1 \%$ of urinary retention, and this occurred in patients under general anaesthesia or intravenous sedation. No retention was reported in patients biopsied under local anaesthetic. ${ }^{47,48,50}$

\section{Camprobe}

CAMPROBE is another method of LA freehand biopsy. This is similar to a co-axial needle but it includes an integrated needle for delivering the local anaesthetic to the periprostatic tissue and the perineal access needle is designed with a funnel to facilitate placement of the biopsy needle within the access needle more safely. With a range of 10-12 cores for systematic biopsy, it demonstrates good tolerability, with low pain and superiority to TRUS biopsy. ${ }^{51}$ Although a small sample size (40 patients) has been used to assess it, it shows good results with $68 \%$ overall cancer and $47 \%$ CSD detection rate. However, this was in a patient population with a known diagnosis of prostate cancer on Active Surveillance, so one might have expected an overall higher cancer detection rate. $^{52}$ The CAMPROBE device is not fixed in line with 
the US probe and so is similar to freehand biopsies and, as demonstrated by Ristau et al., the fan-like technique used in the study is inferior to the sector method. ${ }^{53}$

\section{Optimum Number of Biopsy Cores for Targeted Biopsy}

Regarding the number of cores for targeted biopsy, there seems to be no consensus in the literature. Hansen et al.'s multi-centre study of MRI Fusion and Cognitive Fusion TP biopsies showed the detection of CSD (defined as Gleason Score $\geq 7$ ) increased with median 4-core fusion TP biopsy cores used in one centre and even more with 4 cognitive targeted TP biopsy used in another centre. However, this could be a discrepancy between different centres and not purely the number of cores. ${ }^{32}$ Currently there is literature discussing the number of target cores only using the transrectal approach so it is of great importance that more research is carried out to investigate the minimum number to detect CSD with TP biopsy. There is no real consensus on the number of targeted biopsies that should be taken according to PIRAD score or lesion size. However, Ploussard et al. have examined this methodically in a cohort of patients undergoing fusion targeted biopsies followed by radical prostatectomy and concluded that at least 4 targeted cores should be taken in PIRADS 3 score lesions whereas 3 cores seemed enough in PIRADS 4-5 cases to improve Gleason grade prediction and to limit upgrading risk to $30 \%{ }^{54}$

From a practical perspective the recommendation of the authors is that two biopsies is probably the minimum that should be taken from a large lesion $>10 \mathrm{~mm}$, but with smaller lesions of $4-10 \mathrm{~mm}$ it is probably necessary to take more biopsies between 4 and 6 cores to ensure adequate sampling.

\section{Optimum Number of Cores for Systematic TP Biopsy}

The number of systematic cores varies greatly from paper to paper using different methods. Marra et al. reported a statistically significant increase in pain in proportion with the number of cores under local anaesthetic. ${ }^{34}$ Notwithstanding, there are some studies that argue that the number of cores does not have an effect on the levels of pain during the procedure. Demirtas et al. looked at a total of 252 patients comparing MRI-US Fusion to TRUS-guided 12core biopsy and found no significant difference in the VAS score between the two groups. ${ }^{55}$ Increased number of cores also correlates with increased incidence of urinary retention in the case of template biopsy. ${ }^{15}$ In Kum et al., where the
PrecisionPoint was used, there was no difference in pain scores or complications by increasing number of cores, with a number of $\geq 28$ cores being reported as tolerable. ${ }^{48}$ This suggests that the less invasive 2-puncture-access-point system eliminates the factor of pain and complications regarding the number of cores sampled which are directed to the $\mathrm{PZ}$ rather than sampling the $\mathrm{TZ}$.

\section{Systematic vs Targeted Biopsies}

With regards to the dilemma of combining targeted with systematic biopsy instead of performing solely targeted biopsies, Marra et al. using the freehand Esaote Fusion TP biopsy found that $17.4 \%$ of CSD would have been missed by targeted-only biopsy if systematic biopsy was not performed. ${ }^{34}$ This translates to 1 in 5 men with CSD needing an additional systematic biopsy to be diagnosed with prostate cancer. In Kum et al., with PrecisionPoint cognitive targeted TP biopsy, 6 out of 35 cancer cases (17\%) would have been missed without additional systematic biopsy. ${ }^{48}$ There will always be a risk that targetonly biopsies may under-sample the gland; this would be less important if all patients were treated with radical whole gland treatment but with the increasing use of Active Surveillance and less invasive techniques such as focal therapy, additional systematic biopsy remains necessary. What has not been determined is how best this should be done. It is also worth noting these papers were not designed to specifically answer this research question. Neale et al, also using the PrecisionPoint device, reported a similar number of $12 \%$ of CSD missed by target-only biopsy. Since their missed detection was only $4 \%$ for LIKERT 4 and $0 \%$ for LIKERT 5, the pattern suggests there is a decreasing role for additional systematic biopsy as the suspicion of cancer increases. A 4-core targeted biopsy with additional 6 systematic cores in the quadrant where the lesion was found would have detected $97 \%$ of CSD, supporting a target-quadrant systematic approach. Furthermore, without systematic biopsy, cancer in the contralateral quadrants, apart from the visible lesion, cannot be excluded, and diagnosis is heavily reliant on the quality of MRI imaging and reporting; thus, these patients cannot be treated with focal therapy with certainty. ${ }^{39}$

\section{COVID-I 9 and Prostate Biopsies}

The "exit" from TRUS biopsy has become known as the TRexit initiative. ${ }^{56}$ TRexit has never been more pertinent than during the current COVID-19 pandemic where 
COVID-19 patients occupied most of the hospital beds and utilised most of the resources available. At the start of the Covid-19 pandemic, the British Association of Urological Surgeons (BAUS) recommended that both TRUS biopsies and GA procedures be kept to a minimum. ${ }^{57}$ This has had a significant impact on the prostate diagnostic pathway. A survey of 105 centres during the height of the pandemic indicated that LA TP was the preferred method for prostate biopsy by clinicians; $64 \%$ of those centres offering GA TP and $60 \%$ of those offering TRUS biopsies had to stop prostate biopsies, but only $20 \%$ of centres offering LA TP suspended their biopsy service. ${ }^{58}$

The impact of the pandemic on waiting lists following the suspension of routine elective surgery means that the task that lies ahead to recover the NHS elective surgery position is challenging. The pandemic may have provided the opportunity to increase the uptake of LA TP biopsies nationally as a cost-effective alternative to GA TP biopsies, freeing up lists for other elective procedures that were suspended during the pandemic, and accelerate a transition away from TRUS biopsy, providing a better and safer outpatient biopsy procedure and achieve a UK TRexit. The TRexit approach requires the full uptake of mpMRI prior to biopsy in combination with PSAD to avoid biopsies of non CSD, TP biopsy under LA to avoid aerosol-generating intubation, limiting the risk of urosepsis, while providing a well-tolerated and effective prostate biopsy sampling. It is unrealistic to expect that all cases can be done under LA, however, a LA block in conjunction with light sedation can avoid the use of GA. Despite all the adversities of the pandemic, the need for the
TRexit initiative has been accelerated. There had already been a shift towards TP biopsies even before the Covid-19 pandemic: over the last 10 years TRUS biopsy has outnumbered TP biopsy with a 4:1 ratio. However, in the 2 years before the pandemic this moved to a $2: 1$ ratio. $^{59}$ The latest Hospital Episode Statistics data indicates that prostate biopsies reduced by $30 \%$ overall but TP biopsies accounted for nearly $70 \%$ of all biopsies. The "TRexit" Initiative to transition from TRUS to LA TP seems increasingly achievable (Figure 4), the only question is when.

\section{Conclusion}

Prostate biopsy techniques have evolved into a highly useful and effective diagnostic tool for prostate cancer. Advances such as the Ginsburg Protocol refining the systematic TP biopsy technique and the breakthrough of freehand methods and transperineal access systems revolutionising the way biopsies are performed in an outpatient setting under LA are driving a move towards TRexit across the UK. Cancer detection rates are very acceptable, and the morbidity is low; however, there is still a lot of research required to determine the optimum number of targeted cores and to establish a reproducible strategy for systematic biopsies in patients with equivocal MRI findings. The increasing interest in MRI targeted biopsies, both cognitive and MRI US fusion, is improving the detection of clinically significant disease and reducing the detection of insignificant disease but there is a paucity of comparative data between TRUS MRI Fusion vs TP MRI Fusion techniques. A recent systematic review indicates that most

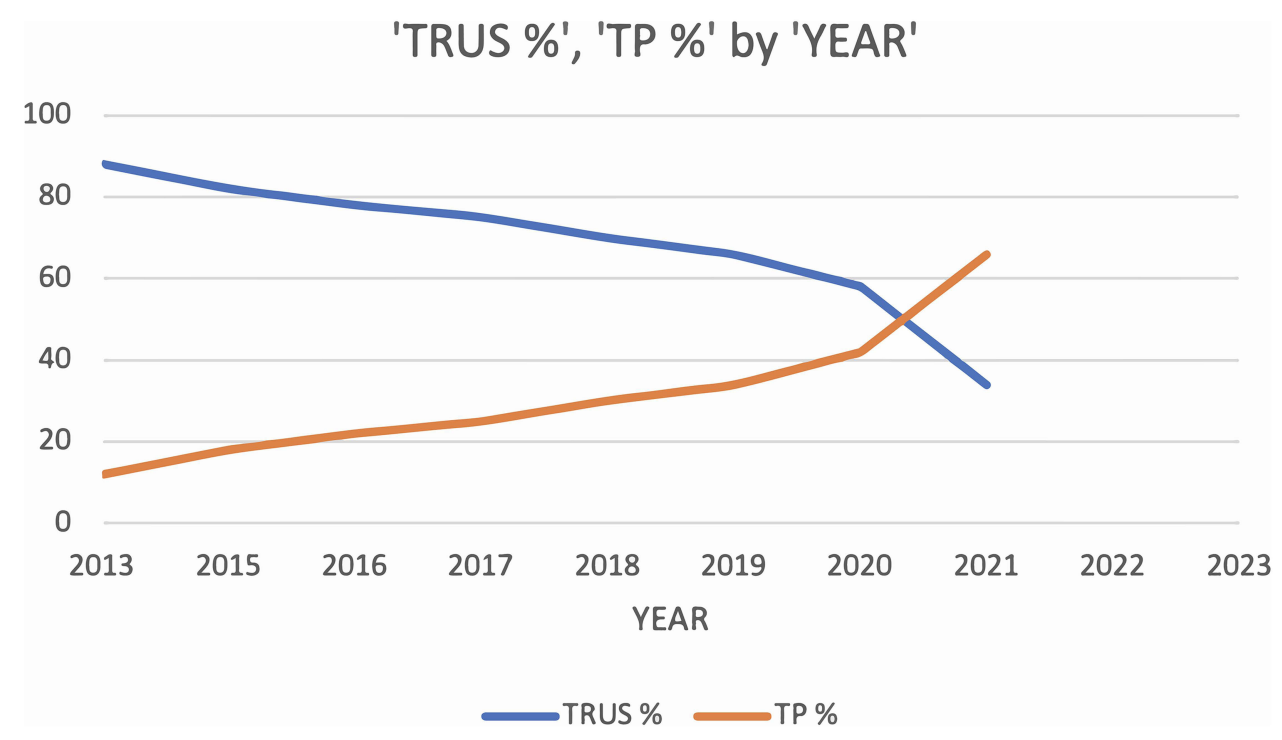

Figure 4 Percentage of TRUS biopsy vs TP biopsy by year 2014-202I (R Popert, Guy's \& St Thomas' Hospital, Personal Communication, June 202I). 
evaluations of TRUS vs TP are comparative cohort studies, mainly because randomized controlled trials are considered inappropriate because of the potential risk of PBRS from TRUS biopsy. ${ }^{60}$ The suggestion is that the TP approach, even with fusion or targeted biopsies, provides better detection of clinically significant disease, anterior tumours and fewer complications, than TRUS; it is a safer biopsy and better fit for a post Covid future.

\section{Acknowledgments}

The Prostate Cancer Specialist Nurses Jonah Rusere and Grace Zisengwe, and Urology Outpatients Lead Nursing Sister Thomasia Azavedo have been instrumental in contributing to the author's clinical work towards TRexit and the smooth running of the prostate diagnostic pathway.

\section{Disclosure}

Rick Popert is a Fellow on the NHS Innovation Accelerator. He receives honoraria for teaching and training from BXTAccelyon, the distributor of the PrecisionPoint Transperineal (TP) Access System. The other authors have nothing to disclose.

\section{References}

1. Marenco J, Kasivisvanathan V, Emberton M. New standards in prostate biopsy. Arch Esp Urol. 2019;72(2):142-149.

2. Ortner G, Tzanaki E, Rai BP, Nagele U, Tokas T. Transperineal prostate biopsy: the modern gold standard to prostate cancer diagnosis. Turk J Urol. 2021;47(1):S19-S26. doi:10.5152/tud.2020.20358

3. Simson N, Rusere J, Stroman L, Popert R. Never mind Brexit, it's time to celebrate 'TRexit'. Trends Urol Men's Health. 2019;10(3):26. doi:10.1002/tre. 696

4. Hodge KK, McNeal JE, Terris MK, Stamey TA. Random systematic versus directed ultrasound guided transrectal core biopsies of the prostate. J Urol. 1989;142(1):71-74; discussion 74-75. doi:10.1016/ S0022-5347(17)38664-0

5. Presti JC Jr. Chang JJ, Bhargava V, Shinohara K. The optimal systematic prostate biopsy scheme should include 8 rather than 6 biopsies: results of a prospective clinical trial. J Urol. 2000;163(1):163-166; discussion 166-167. doi:10.1016/S0022-5347(05)67995-5

6. Moe A, Hayne D. Transrectal ultrasound biopsy of the prostate: does it still have a role in prostate cancer diagnosis? Transl Androl Urol. 2020;9(6):3018-3024. doi:10.21037/tau.2019.09.37

7. Demura T, Hioka T, Furuno T, et al. Differences in tumor core distribution between palpable and nonpalpable prostate tumors in patients diagnosed using extensive transperineal ultrasound-guided template prostate biopsy. Cancer. 2005;103(9):1826-1832. doi:10.1002/cncr.21020

8. Ficarra V, Martignoni G, Novella G, et al. Needle core length is a quality indicator of systematic transperineal prostate biopsy. Eur Urol. 2006;50(2):266-271. doi:10.1016/j.eururo.2005.12.057

9. Huang GL, Kang CH, Lee WC, Chiang PH. Comparisons of cancer detection rate and complications between transrectal and transperineal prostate biopsy approaches - a single center preliminary study. $B M C$ Urol. 2019;19(1):101. doi:10.1186/s12894-019-0539-4
10. Newman T, Stroman L, Hadjipavlou M, et al. A switch from transrectal biopsy to cognitive freehand transperineal prostate biopsy can reduce sepsis where targeted antibiotics for resistant rectal flora have failed. Eur Urol. 2020;19(2):312.

11. Tsivian M, Kimura M, Sun L, Mouraviev V, Mayes JM, Polascik TJ. Predicting unilateral prostate cancer on routine diagnostic biopsy: sextant vs extended. BJU Int. 2010;105(8):1089-1092. doi:10.1111/ j.1464-410X.2009.08904.x

12. Nakai Y, Tanaka N, Maesaka F, Miyake M, Fujii T, Fujimoto K. Evaluating the optimal technique of transperineal template-guided saturation biopsy in patients requiring repeat prostate biopsy. Int J Urol. 2020;27(12):1157-1158. doi:10.1111/iju.14373

13. Crawford ED, Wilson SS, Torkko KC, et al. Clinical staging of prostate cancer: a computer-simulated study of transperineal prostate biopsy. $B J U I$. 2005;96(7):999-1004. doi:10.1111/j.1464-410X.2005.05801.x

14. Wang F, Ding XF, Xu JN, et al. [Complications of transperineal template-guided prostate mapping biopsy]. Zhonghua Yi Xue Za Zhi. 2019;99(6):428-431. Chinese.

15. Kum F, Jones A, Nigam R. Factors influencing urinary retention after transperineal template biopsy of the prostate: outcomes from a regional cancer centre. World J Urol. 2018;37(2):337-342. doi:10.1007/s00345-018-2390-8

16. Kuru TH, Wadhwa K, Chang RT, et al. Definitions of terms, processes and a minimum dataset for transperineal prostate biopsies: a standardization approach of the Ginsburg study group for enhanced prostate diagnostics. BJUI. 2013;112(5):568-577. doi:10.1111/bju.12132

17. Challacombe B, Dasgupta P, Patel U, Amoroso P, Kirby R. Recognizing and managing the complications of prostate biopsy. BJU Int. 2011;108 (8):1233-1234. doi:10.1111/j.1464-410X.2011.10621.x

18. Vyas L, Acher P, Kinsella J, et al. Indications, results and safety profile of transperineal sector biopsies (TPSB) of the prostate: a single centre experience of 634 cases. BJU Int. 2014;114 (1):32-37. doi:10.1111/bju.12282

19. Hansen N, Patruno G, Wadhwa K, et al. Magnetic Resonance and Ultrasound Image Fusion supported transperineal prostate biopsy using the ginsburg protocol: technique, learning points, and biopsy results. Eur Urol. 2016;70(2):332-340.

20. Eldred-Evans D, Kasivisvanathan V, Khan F, et al. The use of transperineal sector biopsy as a first-line biopsy strategy: a multi-institutional analysis of clinical outcomes and complications. Urol J. 2016;13(5):2849-2855.

21. Stamey TA, Yang N, McNeal JE, Freiha FS, Redwine E. Prostatespecific antigen as a serum marker for adenocarcinoma of the prostate. $N$ Engl J Med. 1987;317(15):909-916. doi:10.1056/ NEJM198710083171501

22. MacAskill F, Lee SM, Eldred-Evans D, et al. Diagnostic value of MRI-based PSA density in predicting transperineal sector-guided prostate biopsy outcomes. Int Urol Nephrol. 2017;49(8):1335-1342. doi:10.1007/s11255-017-1609-8

23. Washino S, Okochi T, Saito K, et al. Combination of prostate imaging reporting and data system (PI-RADS) score and prostate-specific antigen (PSA) density predicts biopsy outcome in prostate biopsy naïve patients. BJU Int. 2017;119(2):225-233. doi:10.1111/bju.13465

24. Rico L, Contreras P, Vitagliano G, Pita HR, Ameri C, Blas L. Value of prostate-specific antigen density in negative or equivocal lesions on multiparametric magnetic resonance imaging. Turk $J$ Urol. 2020;46(5):367-372. doi:10.5152/tud.2020.20111

25. Sonmez G, Tombul ST, Demirtas T, Demirtas A. Clinical factors for predicting malignancy in patients with PSA $<10 \mathrm{ng} / \mathrm{mL}$ and PIRADS 3 lesions. Asia Pac J Clin Oncol. 2021;17(2):e94-e99.

26. Pokorny M, Kua B, Esler R, et al. MRI-guided in-bore biopsy for prostate cancer: what does the evidence say? A case series of 554 patients and a review of the current literature. World J Urol. 2019;37 (7):1263-1279. doi:10.1007/s00345-018-2497-y 
27. D'Agostino D, Romagnoli D, Giampaoli M, et al. "In-Bore" MRIguided prostate biopsy for prostate cancer diagnosis: results from 140 consecutive patients. Curr Urol. 2020;14(1):22-31. doi:10.1159/ 000499264

28. Porpiglia F, Manfredi M, Mele F, et al. Diagnostic pathway with multiparametric magnetic resonance imaging versus standard pathway: results from a randomized prospective study in biopsy-naïve patients with suspected prostate cancer. Eur Urol. 2017;72 (2):282-288. doi:10.1016/j.eururo.2016.08.041

29. Ahmed HU, El-Shater Bosaily A, Brown LC, et al. Diagnostic accuracy of multi-parametric MRI and TRUS biopsy in prostate cancer (PROMIS): a paired validating confirmatory study. Lancet. 2017;389(10071):815-822. doi:10.1016/S0140-6736(16)32401-1

30. Ahmed HU, Kirkham A, Arya M, et al. Is it time to consider a role for MRI before prostate biopsy? Nat Rev Clin Oncol. 2009;6 (4):197-206. doi:10.1038/nrclinonc.2009.18

31. Kasivisvanathan V, Rannikko AS, Borghi M, et al. MRI-targeted or standard biopsy for prostate-cancer diagnosis. $N$ Engl J Med. 2018;378(19):1767-1777. doi:10.1056/NEJMoa1801993

32. Hansen NL, Barrett T, Kesch C, et al. Multicentre evaluation of magnetic resonance imaging supported transperineal prostate biopsy in biopsy-naïve men with suspicion of prostate cancer. BJU Int. 2018;122(1):40-49. doi:10.1111/bju.14049

33. Martorana E, Pirola GM, Aisa MC, et al. Prostate MRI and transperineal TRUS/MRI fusion biopsy for prostate cancer detection: clinical practice updates. Turk J Urol. 2019;45(4):237-244. doi:10.5152/ tud.2019.19106

34. Marra G, Marquis A, Tappero S, et al. Transperineal free-hand mpMRI fusion-targeted biopsies under local anesthesia: technique and feasibility from a single-center prospective study. Urology. 2020;140:122-131. doi:10.1016/j.urology.2019.11.078

35. Marra G, Zhuang J, Beltrami M, et al. Transperineal freehand multiparametric MRI fusion targeted biopsies under local anaesthesia for prostate cancer diagnosis: a multicentre prospective study of 1014 cases. BJUI. 2021;127(1):122-130. doi:10.1111/bju.15121

36. Gayet M, Beerlage HP, Schrier BP, Mulders PFA, Wijkstra H. The value of magnetic resonance imaging and ultrasonography (MRI/US)fusion biopsy platforms in prostate cancer detection: a systematic review. BJUI. 2015;117(3):392-400. doi:10.1111/bju.13247

37. Watts KL, Frechette L, Muller B, et al. Systematic review and meta-analysis comparing cognitive vs. image-guided fusion prostate biopsy for the detection of prostate cancer. Urol Oncol. 2020;38 (9):734.e719-734.e725. doi:10.1016/j.urolonc.2020.03.020

38. Zhang Q, Wang W, Zhang B, et al. Comparison of free-hand transperineal mpMRI/TRUS fusion-guided biopsy with transperineal 12-core systematic biopsy for the diagnosis of prostate cancer: a single-center prospective study in China. Int Urol Nephrol. 2017;49 (3):439-448. doi:10.1007/s11255-016-1484-8

39. Neale A, Stroman L, Kum F, et al. Targeted and systematic cognitive freehand-guided transperineal biopsy: is there still a role for systematic biopsy? BJUI. 2020;126(2):280-285. doi:10.1111/bju.15092

40. Oderda M, Faletti R, Battisti G, et al. Prostate cancer detection rate with koelis fusion biopsies versus cognitive biopsies: a comparative Study. Urol Int. 2017;97(2):230-237. doi:10.1159/000445524

41. Braeckman J, Autier P, Garbar C, et al. Computer-aided ultrasonography (HistoScanning): a novel technology for locating and characterizing prostate cancer. BJU Int. 2008;101(3):293-298. doi:10.1111/j.1464-410X.2007.07232.x

42. Simmons LA, Autier P, Zát'ura F, et al. Detection, localisation and characterisation of prostate cancer by prostate HistoScanning(TM). BJU Int. 2012;110(1):28-35. doi:10.1111/j.1464-410X.2011.10734.x

43. Sivaraman A, Sanchez-Salas R, Barret E, et al. Prostate histoscanning true targeting guided prostate biopsy: initial clinical experience. World J Urol. 2015;33(10):1475-1479. doi:10.1007/s00345-014-1434-y
44. Schiffmann J, Tennstedt P, Fischer J, et al. Does HistoScanning ${ }^{\text {TM }}$ predict positive results in prostate biopsy? A retrospective analysis of 1188 sextants of the prostate. World J Urol. 2014;32(4):925-930. doi:10.1007/s00345-014-1330-5

45. Grey ADR, Connor MJ, Tam J, Loch T. Can transrectal prostate ultrasound compete with multiparametric MRI in the detection of clinically significant prostate cancer? Transl Androl Urol. 2020;9 (3):1492-1500. doi:10.21037/tau.2020.02.26

46. Glybochko PV, Alyaev YG, Amosov AV, et al. Evaluation of prostate histoscanning as a method for targeted biopsy in routine practice. Eur Urol Focus. 2019;5(2):179-185. doi:10.1016/j.euf.2017.07.001

47. Stefanova V, Buckley R, Flax S, et al. Transperineal prostate biopsies using local anesthesia: experience with 1287 patients. prostate cancer detection rate, complications and patient tolerability. $J$ Urol. 2019;201(6):1121-1126. doi:10.1097/JU.0000000000000156

48. Kum F, Elhage O, Maliyil J, et al. Initial outcomes of local anaesthetic freehand transperineal prostate biopsies in the outpatient setting. BJUI. 2020;125(2):244-252. doi:10.1111/bju.14620

49. Perineologic. Introducing the PrecisionPoint ${ }^{\mathrm{TM}}$ Transperineal Access System [Internet]. [place unknown]. [publisher unknown]; 2021 [cited 2021 Feb 28]. Available from: https://perineologic.com/preci sionpoint/. Accessed July 2, 2021.

50. Gorin MA, Meyer AR, Zimmerman M, et al. Transperineal prostate biopsy with cognitive magnetic resonance imaging/biplanar ultrasound fusion: description of technique and early results. World J Urol. 2020;38(8):1943-1949. doi:10.1007/s00345-019-02992-4

51. Thurtle D, Starling L, Leonard K, Stone T, Gnanapragasam VJ. Improving the safety and tolerability of local anaesthetic outpatient transperineal prostate biopsies: a pilot study of the CAMbridge PROstate Biopsy (CAMPROBE) method. J Clin Urol. 2018;11 (3):192-199. doi:10.1177/2051415818762683

52. Gnanapragasam VJ, Leonard K, Sut M, et al. Multicentre clinical evaluation of the safety and performance of a simple transperineal access system for prostate biopsies for suspected prostate cancer: the CAMbridge PROstate Biopsy DevicE (CamPROBE) study. J Clin Urol. 2020;13(5):364-370. doi:10.1177/2051415820932773

53. Ristau BT, Allaway M, Cendo D, et al. Free-hand transperineal prostate biopsy provides acceptable cancer detection and minimizes risk of infection: evolving experience with a 10-sector template. Urol Oncol. 2018;36(12):528.e515-528.e520. doi:10.1016/j.urolonc.2018.09.013

54. Ploussard G, Beauval JB, Renard-Penna R, et al. Assessment of the minimal targeted biopsy core number per MRI lesion for improving prostate cancer grading prediction. J Clin Med. 2020;9(1):1. doi: $10.3390 / \mathrm{jcm} 9010225$

55. Demirtaş A, Sönmez G, Tombul ŞT, Demirtaş T. Comparison of pain levels in fusion prostate biopsy and standard TRUS-Guided biopsy. Int Braz J Urol. 2020;46(4):557-562. doi:10.1590/s1677-5538. ibju.2019.0154

56. Grummet J, Gorin MA, Popert R, et al. "TREXIT 2020": why the time to abandon transrectal prostate biopsy starts now. Prostate Cancer Prostatic Dis. 2020;23(1):62-65. doi:10.1038/s41391-020-0204-8

57. COVID-19 strategy for the interim management of prostate cancer. British Association of Urology Section of Oncology; 2020.

58. Stroman L, Cathcart P, Lamb A, et al. A cross-section of UK prostate cancer diagnostics during the coronavirus disease 2019 (COVID-19) era - a shifting paradigm? BJUI. 2021;127(1):30-34. doi:10.1111/bju.15259

59. Tamhankar AS, El-Taji O, Vasdev N, et al. The clinical and financial implications of a decade of prostate biopsies in the NHS: analysis of hospital episode statistics data 2008-2019. BJUI. 2020;126 (1):133-141. doi:10.1111/bju.15062

60. Rai BP, Mayerhofer C, Somani BK, Kallidonis P, Nagele U, Tokas T. Magnetic resonance imaging/ultrasound fusion-guided transperineal versus magnetic resonance imaging/ultrasound fusion-guided transrectal prostate biopsy-A Systematic Review. Eur Urol Oncol. 2021. doi:10.1016/j.euo.2020.12.012 


\section{Publish your work in this journal}

Research and Reports in Urology is an international, peer-reviewed, open access journal publishing original research, reports, editorials, reviews and commentaries on all aspects of adult and pediatric urology in the clinic and laboratory including the following topics: Pathology, pathophysiology of urological disease; Investigation and treatment of urological disease; Pharmacology of drugs used for the treatment of urological disease. The manuscript management system is completely online and includes a very quick and fair peer-review system, which is all easy to use. Visit http://www.dovepress.com/ testimonials.php to read real quotes from published authors.

Submit your manuscript here: https://www.dovepress.com/research-and-reports-in-urology-journal 